\title{
Comparative assessment of pharmacokinetics,

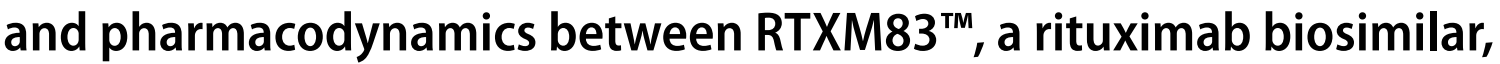 and rituximab in diffuse large B-cell lymphoma patients: a population PK model approach
}

\author{
Myrna Candelaria ${ }^{1}$ - Derlis Gonzalez ${ }^{2} \cdot$ Francisco Javier Fernández Gómez ${ }^{3}$ Alexandra Paravisini ${ }^{3}$. \\ Ana Del Campo García ${ }^{3} \cdot$ Luis Pérez $^{3} \cdot$ Bernardo Miguel-Lillo $^{4}$. Susana Millán ${ }^{3}$
}

Received: 28 September 2017 / Accepted: 17 January 2018 / Published online: 24 January 2018

(c) The Author(s) 2018. This article is an open access publication

\begin{abstract}
Purpose The main objective was to quantify any potential differences in pharmacokinetic (PK) parameters (AUC and $C_{\max }$ ) between RTXM83, a proposed rituximab biosimilar, and its reference product, using a population PK model approach.

Methods Rituximab PK and PD data were obtained from a randomized, double-blind, phase III clinical study (RTXM83AC-01-11) in patients with diffuse large B-cell lymphoma (DLBCL) that received $375 \mathrm{mg} / \mathrm{m}^{2}$ intravenous RTXM83 or its reference product with CHOP regimen, every 3 weeks, for six cycles. Rituximab levels were quantified by Meso Scale Discovery assay. PK analysis was performed using NONMEM 7.3.0. The effect of disease and patient covariates on RXTM83 PK was investigated. Model was evaluated using visual predictive check and non-parametric bootstrap.

Results In total, 251 DLBCL patients (127 and 124 in RXTM83-CHOP and rituximab-CHOP arms, respectively) and 5341 serum concentrations (2703 for RXTM83 and 2638 for rituximab, respectively) were available for the population PK analysis. The volume of distribution of the central compartment $\left(V_{1}\right)$ and clearance of RXTM83 were estimated at $3.19 \mathrm{~L}$ and $12.5 \mathrm{~mL} / \mathrm{h}$, respectively. Body surface area allowed to explain the interindividual variability for $V_{1}$. A statistical analysis showed that systemic exposure (AUC and $C_{\max }$ ) of RTXM83 was similar to rituximab. The $90 \%$ confidence intervals for all pairwise comparisons were within the predefined bioequivalence interval of 0.80-1.25. PD similarity of B-cell depletion and recovery was also observed.

Conclusions The time course of RTXM83 was well characterized by the model developed. The systemic exposure of RTXM83 and its associated variability were similar to those for rituximab reference in DLBCL patients, demonstrating PK similarity. The PD similarity of RTXM83 and rituximab reference product was also demonstrated.
\end{abstract}

Keywords Rituximab · RTXM83 - Population pharmacokinetics · Biosimilar · Diffuse large B-cell lymphoma ·

Pharmacodynamics $\cdot$ ADA assessment

Francisco Javier Fernández Gómez

javierfergo@outlook.com

1 Instituto Nacional de Cancerología, Av. San Fernando No. 22, Tlalpan, Sección XVI, Mexico, DF, Mexico

2 Instituto Privado de Hematología e Investigación Clínica (IPHIC), Mariano Roque Alonso 1450, Asunción, Paraguay

3 mAbxience Research S.L., Manuel Pombo Angulo 28, 28050 Madrid, Spain

4 SGS Exprimo NV, Mechelen, Belgium

\section{Introduction}

Rituximab (MabThera ${ }^{\circledR}$ or Rituxan ${ }^{\circledR}$ ), a chimeric antiCD20 human immunoglobulin G1 monoclonal antibody, has revolutionized the treatment of various types of non-Hodgkin's lymphoma (NHL), chronic lymphocytic leukemia, and is also approved for rheumatoid arthritis and other autoimmune diseases. Unfortunately, access to rituximab is limited in many areas of the world mainly due to its cost. Rituximab biosimilars can be used as safely and effectively in all their approved indications but at a lower cost, thereby improving patient access [1]. 
Biosimilarity is confirmed when the biological product is highly similar to the reference product notwithstanding minor differences in clinically inactive components, and there are no clinically meaningful differences between the biological product and the reference product in terms of the safety, purity, and potency of the product [1]. The approval process for biosimilars includes an assessment of the pharmacokinetics (PK) and pharmacodynamics (PD) if applicable, in the most sensitive population to demonstrate that there are no major differences between reference and biosimilar products in accordance with the regulatory requirements [2, 3]. These PK/PD studies support a demonstration of biosimilarity with the assumption that similar exposure (and PD response) provides similar efficacy and safety. Healthy volunteers are the most sensitive population for PK studies [4]; however, a PK study with rituximab in healthy volunteers is not ethically acceptable due to safety risks associated with rituximab exposure. Hence, a sensitive population of patients should be chosen to assess PK comparability of a rituximab biosimilar.

RTXM83 is a rituximab biosimilar developed by $\mathrm{mAbx}$ ience Research S.L. that has demonstrated sufficient similarity to the reference medicinal product [5]. The PK and PD comparability of RTXM83 and the reference medicinal product were assessed as secondary endpoints in a randomized, double-blind, phase III clinical study in a population of selected diffuse large B-cell lymphoma (DLBCL) patients (NCT02268045). DLBCL was chosen as the most "sensitive and homogeneous" population, because it represents the best characterized disease from the approved indications for rituximab, with the most uniform population and a higher level of clinical response to rituximab and better prognosis [6]. Likewise, the schedule of treatment for DLBCL (six cycles with one dose every 21 days per cycle) provides a considerable number of PK measurements from a high population of patients to have a complete PK profile allowing the comparability with the reference product.

The PK similarity between RTXM83 and the rituximab reference product was assessed in the study by comparing the predicted serum concentrations over time, derived from a population PK model. Unlike the non-compartmental approach, population modelling allows the use of sparse data to accurately calculate PK parameters. Furthermore, the population approach allows to quantify the interproduct and intersubject variability on PK parameters and to identify covariate factors (demographic, pathophysiological, environmental, or concomitant drugs) that influence drug availability [4].

The main PK endpoints in the study, the area under the concentration-time curve (AUC) and the maximum serum concentration $\left(C_{\max }\right)$, were calculated for the two compounds using the individual PK parameters estimated by the population PK model and were subsequently evaluated by traditional bioequivalence methods in accordance with regulatory guidelines on how to address PK similarity [2, 3]. In addition, the levels of CD20+ and CD19+ B-cells in peripheral blood were assessed in the study for the PD evaluation. CD19+ was used as surrogate marker in the population PK model in patients with circulating rituximab, since its expression mirrors CD20 expression [7]. Finally, immunogenicity based on anti-drug antibody (ADA) detection was analysed to assess the potential impact on PK profile.

Our aim is to present this unique population PK model with rituximab biosimilar in a high sample size of patients and demonstrate how well describes the PK similarity between a rituximab biosimilar and its reference medicinal product. In addition, here, we describe the good correlation of PK profile with the PD kinetics observed in the study.

\section{Patients and methods}

\section{Patients}

The study (RTXM83-AC-01-11) was a randomized, double-blind, phase III study comparing biosimilar rituximab (RTXM83) plus CHOP chemotherapy (RTXM83-CHOP) with MabThera ${ }^{\circledR}$ plus CHOP (rituximab-CHOP) in the firstline treatment of patients with DLBCL. Patients were aged $18-65$ years and had a stage II-IV and bulky disease by Ann arbor classification, ECOG performance status of $0-2$, and IPI 0-1. Subjects received RTXM83 or rituximab reference product at a dose of $375 \mathrm{mg} / \mathrm{m}^{2}$ on day 1 of each 3-week cycle in combination with CHOP chemotherapy (cyclophosphamide, doxorubicin, vincristine, and prednisone), from one to six treatment cycles. The primary objective was to determine if the response rate with RTXM83-CHOP was non-inferior to that with rituximab-CHOP. Secondary objectives were to demonstrate comparable PK, PD (decreased CD20+ and CD19+ B-cells), and immunogenicity (ADA), as well as to evaluate safety of RTXM83 vs its reference product when administered with CHOP (NCT02268045).

The study was conducted in accordance with Good Clinical Practice guidelines and the principles of the Declaration of Helsinki. The study was approved by the corresponding ethics committees, and informed consent was obtained from all subjects after they had been advised of the investigational nature of the study and its potential risks and benefits, and before undergoing any study procedure.

\section{Sampling and bioanalytical methods}

Blood samples for the PK assessment were collected at prespecified intervals to obtain PK profiles for the determination of single-dose and steady-state PK at cycle 6. These PK samples were collected in all patients at the following time 
points: cycle 1: Day 1 (pre-dose, mid-infusion and at the end of infusion [EOI]), Day 8 (EOI) and Day 15 (EOI); cycles 2 and 4: Day 1 (pre-dose and EOI), Day 8 (EOI) and Day 15 (EOI); cycles 3 and 5: Day 1 (pre-dose and EOI); and cycle 6 (last dose): Day 1 (pre-dose and EOI), and at EOI on Day 8, Day 15 and Day 21.

A validated quantitative immunoassay was used for the determination of either RTXM83 or rituximab reference product in the human serum from samples collected from patients in the RTXM83-AC-01-11 study. The assay comprised a first incubation of the diluted samples to allow rituximab (RTXM83 or reference product) to bind to biotinylated-anti-rituximab. After incubation overnight, samples were added to the streptavidin-coated plate. The anti-rituximab-biotin complex binds to the streptavidin-coated wells, allowing any unbound material to be washed away; Sulfo Tag (sTag)-anti-rituximab was added and binds to RTXM83 or the rituximab reference product. Read buffer containing tripolyamine was added and the sTag associated with antirituximab (RTXM83/ reference product) produced a chemiluminescent signal when an electrical voltage is applied. The luminescence signal was directly proportional to the concentration of drug present. The data were generated using Meso Scale Discovery (MSD; electrochemiluminescence [ECL]) platform and processed using Softmax Pro by Covance Laboratories Ltd., Harrogate, United Kingdom.

Ten standard calibrators from 20 to $4000 \mathrm{ng} / \mathrm{mL}$ and five quality controls, with concentrations of $40,120,700$, 2200 , and $3000 \mathrm{ng} / \mathrm{L}$, were used in the validation process. The lower limit of quantification (LLOQ) for RTXM83 and rituximab was $40.0 \mathrm{ng} / \mathrm{mL}$, and the mean overall coefficient of variation was less than $20 \%$ across the range of concentrations, which included up to $3000.00 \mathrm{ng} / \mathrm{mL}$.

Blood samples for CD20+ and CD19+ B-cell counts (PD analysis) were collected at the following time points: cycle 1 at Day 1 (pre-dose and at the EOI), Day 8 (EOI) and Day 15 (EOI), cycles 2 and 4 at Day 1(pre-dose), cycle 6 at Day 1 (pre-dose) and Day 21 (EOI); and during the follow-up (FU) period (3 (FU1), 6 (FU2) and 9 (FU3) months after last dose of chemotherapy). Blood samples were analysed using a flow cytometry procedure. The median percent changes from baseline in blood of CD20+ and CD19+ B-cell following the administration of reference product antibodies and anti-RTXM83 co-administered with CHOP in cycles 1-6 (including last FU observation) were plotted by treatment and cycle/timepoint.

Blood samples for ADA analysis were collected at baseline (pre-dose), in cycle 5 Day 1, cycle 6 Day 21, and during the follow-up period if available (3 and 9 months after last dose of treatment). A single validated ECL method (using MSD platform) was used to test samples for both antirituximab reference product antibodies, and anti-RTXM83 antibodies.
The immune response after rituximab administration was evaluated by a multi-tiered approach which compromises an immunogenicity assay for the screening, confirmation, and titration. All samples were subjected to an initial screening assay, and those falling above a specific pre-determined screening cut-point were deemed to be positive, and tested in the confirmation assay. For the confirmatory assay, any screened positive samples will be splitted and preincubated with RTXM83 or the rituximab reference product (dependant on dosing) or buffer prior to analysis on the MSD. A reduction in signal upon addition of RTXM83 or the rituximab reference product greater than the validated inhibitory cut-point confirms the specificity of antibody response as anti-RTXM83 or anti-rituximab reference product, following the recommendations made by Shankar et al. [8]. Samples which are confirmed as being specific against rituximab reference product or RTXM83 will have their relative titre assessed.

\section{Population pharmacokinetic analysis}

The following strategy was used to develop the final model. First, the structural PK model was developed. Second, a heterogeneity model, including significant covariates, was then identified and incorporated in the model, and the final model was then evaluated by complementary methods for assess robustness.

\section{Software}

The analyses and simulations were performed using NONMEM Version 7.3.0 software [9], supplemented with the PsN toolkit version 4.4.8 [10], and were further processed using R Version 3.2.0 [11]. NONMEM data sets were prepared using SAS 9.4 (SAS Institute Inc., Cary, NC, USA). Analyses were performed on an Intel Xeon-based server running Linux (Centos release 5.5, 64 bit) and an Intel Core i7-4702HQ CPU-based personal computer, running under Windows 8.1 (64 bit). NONMEM runs were performed using the Intel Visual Fortran compiler, Version 11.1.056 (64 bit).

\section{Structural PK model}

Rituximab PK has been extensively described in the literature [12-14]. Based on the published data, an open two-compartment PK model with linear distribution and linear elimination from the central compartment was used to describe the time course of rituximab in DLBCL subjects. This model was fitted to the data available and the typical value of clearance (CL), central volume of distribution $\left(V_{1}\right)$, intercompartmental flow between central and peripheral compartment $(Q)$, and volume of distribution of 
the peripheral compartment $\left(V_{2}\right)$ were obtained. The NONMEM model fitting used the First Order Conditional Estimation method (FOCE) with the INTERACTION option.

\section{Statistical model}

Visual inspection of individual concentration-time profiles suggested that each PK parameter varied between patients. Patients received rituximab or RTXMB83 in more than one occasion, and PK data available evidenced random within-subject variability. Consequently, between-subject variability (interindividual, IIV) and within-subject (interoccasion, IOV) variability in a PK parameter were included in the model:

$P_{i, k}=P_{\mathrm{TV}} \times\left(e^{\eta_{i}+\tau_{k}}\right)$,

where $P_{i, k}$ is the individual PK parameter value for the $i$ th individual and $k$ th occasion, $P_{\mathrm{TV}}$ is the typical population estimate of the PK parameter, and $\eta_{p j}$ and $\zeta_{\mathrm{pk}}$ are a normally distributed between- and within-subject random variable with zero-mean and variance $\omega_{p}{ }^{2}$ and $\pi_{p}{ }^{2}$, respectively. The magnitudes of IIV and interoccasion variability (IOV) were expressed as coefficients of variations (CVs). Two occasions were defined: one for the first cycle of treatment and two for the rest of cycles. Residual variability was modelled using a combined additive and proportional error model. The need for both residual error terms was evaluated during model development based on whether the parameter estimates supported that both terms meaningfully contributed to variability.

\section{Model selection criteria}

The improvement in the fit obtained for each model was assessed in several ways. First, the resulting NONMEM generated minimum value of the objective function (MFOV) after fitting the models evaluated was used to perform the likelihood ratio test. This test is based on the change in the minimum value of the objective function ( $\triangle \mathrm{MVOF}$ ), which is equal (up to a constant) to minus twice the log-likelihood of the data and is asymptotically distributed like Chi-square with the degrees of freedom equal to the number of parameters added to the model. For hierarchical models, a $\triangle \mathrm{MVOF}$ of 7.88 was required to reach statistical significance $(p=0.005)$ for the addition of one fixed effect. In addition, the improvement in the model fit by including covariates into the population PK model was assessed by the reduction in the IIV, IOV, and residual variability, the precision and the correlation in parameter estimates; and finally, the examination of diagnostic plots and shrinkage.

\section{Covariate analysis}

The covariates explored as possible sources of IIV in RTXM83 PK were age, weight, body surface area (BSA), sex, CD19+ cell count (surrogate marker), bone marrow involvement, performance status (PS), bulky disease, extranodal lesions, and IPI score. Once the structural model was identified, empirical Bayes estimates of the interindividual random effects were computed. The covariate screening was guided by graphical assessment between the Bayesian estimates of interindividual random effects and the covariates. Only covariates with statistically significant $(p<0.01)$ and potentially clinically relevant $\left(r^{2}>0.2\right)$ effect on model parameters in the screening analysis were further tested in NONMEM. Those covariates identified by the screening analysis as having a potential influence on a particular parameter were statistically tested one by one for inclusion in the population PK model (forward inclusion, $p<0.05$ ). Categorical covariates were incorporated into the model as index variable, whereas continuous covariates were evaluated using power equations after centering on the median. Covariates with statistically significant effects were incorporated into the population model simultaneously, and subsequently, the covariate screening process was repeated. A full model was identified when no further covariate additions were possible. Then, the relative contribution of each covariate to the model was evaluated using backward elimination, $(p<0.01)$ [15]. All non-significant effects on PK parameters were removed from the model and the covariate model was obtained.

\section{Model qualification}

Three complementary methods were employed to evaluate the model developed: a non-parametric bootstrap [16], normalized prediction distribution errors (NPDEs) [17, 18], and a visual predictive check (VPC) [19]. Non-parametric bootstrap was conducted to assess parameter precision and derive confidence intervals (CI). For this, 1000 data sets were generated by randomly sampling with replacement subjects from the original data set. Model parameters were reestimated on each bootstrap data sets and summary statistics were derived. The 5th and 95th percentiles of the bootstrap parameter distribution constitute the $90 \%$ CI. The distribution of the NPDE was explored by a frequency histogram. Finally, the VPC looks at the model's ability to simulate the same data that have been used for the model development. Serum concentrations of rituximab and RTXM83 were simulated 500 times using the dose and covariate data as well as the same sampling schedule from the subjects that were used in the model development data set. The 5th, $50^{\text {th }}$, and 95th percentiles of the predictions and observations were derived and plotted against time. 


\section{Pharmacokinetic similarity assessment}

The assessment of the PK similarity between RTXM83 and rituximab reference product was based on statistical evaluation, and the average equivalence statistical approach was used. Therefore, the $90 \% \mathrm{CI}$ for the ratio between the geometric means of the $C_{\max }$ and $\mathrm{AUC}_{0-\infty}$ (derived from the population PK parameters) of RTXM83 and the rituximab reference product were calculated at cycle 1 and at cycle 6 (steady state). To conclude PK similarity, the limits for the CI of the ratio should be within the $0.80-1.25$ interval [20, 21].

\section{Results}

\section{Patients}

A total of 251 DLBCL patients were included in the study (127 in RTXM83-CHOP arm and 124 in rituximab-CHOP arm). Patient characteristics at baseline, stratified by arm, are shown in Table 1. All these covariates showed a similar distribution among the two arms, and only RTXM83 arm had a higher median count of CD19+ cells/ $\mu \mathrm{L}$ (89.10 vs 63.35 cell $/ \mu \mathrm{L}$ ). A total of 2703 RTXM83 and 2638 rituximab serum concentrations were included in the analysis data set.

\section{Population pharmacokinetic modelling}

In our model, the time course of RXTM83 serum concentrations in DLBCL patients was described by an open two compartmental disposition model with linear distribution between central and peripheral compartments and linear elimination from the central compartment after i.v. administration as it occurs with the reference medicinal product. The typical volumes of the distribution for central and peripheral compartments were estimated at 3.19 and $4.15 \mathrm{~L}$, respectively, with interindividual variability (IIV) of 14 and $27 \%$, respectively. The apparent volume of distribution at steady state (sum of the volumes of the compartments) was estimated at $3.2 \mathrm{~L}$. The typical rituximab serum clearance was $12.5 \mathrm{~mL} / \mathrm{h}$, with moderate IIV of $25 \%$, whereas the intercompartmental clearance and its associated IIV were estimated at $18.6 \mathrm{~mL} / \mathrm{h}$ and $28 \%$ respectively. The terminal half-life was estimated at 21.6 days.
Table 1 Patient characteristics at baseline, stratified by arm

\begin{tabular}{|c|c|c|c|}
\hline Patient characteristics & $\begin{array}{l}\text { RTXM83-CHOP arm } \\
(n=127,50.6 \%)\end{array}$ & $\begin{array}{l}\text { Rituximab-CHOP arm } \\
(n=124,49.4 \%)\end{array}$ & Total $(n=251)$ \\
\hline \multicolumn{4}{|l|}{ Age (years) } \\
\hline Median (range) & $49.00(18.00-65.00)$ & $52.00(26.00-66.00)$ & $51.00(18.00-66.00)$ \\
\hline \multicolumn{4}{|l|}{ Weight (kg) } \\
\hline Median (range) & $66.00(35.81-137.30)$ & $65.80(33.00-128.00)$ & $66.00(33.00-137.30)$ \\
\hline \multicolumn{4}{|c|}{ Body surface area $\left(\mathrm{BSA} ; \mathrm{m}^{2}\right)$} \\
\hline Median (range) & $1.73(1.23-2.54)$ & $1.71(1.14-2.28)$ & $1.72(1.14-2.54)$ \\
\hline \multicolumn{4}{|l|}{ CD19 (cell/uL) } \\
\hline Median (range) & $89.10(0.02-1436.40)$ & $63.35(0.00-2319.90)$ & $73.15(0.00-2319.90)$ \\
\hline \multicolumn{4}{|l|}{ Sex } \\
\hline Male & $70(55.1 \%)$ & $67(54.0 \%)$ & $137(54.6 \%)$ \\
\hline \multicolumn{4}{|c|}{ Bone marrow involvement } \\
\hline Yes & $6(4.7 \%)$ & $2(1.6 \%)$ & $8(3.2 \%)$ \\
\hline \multicolumn{4}{|l|}{ ECOG PS } \\
\hline 0 & $79(62.2 \%)$ & $75(60.5 \%)$ & $154(61.4 \%)$ \\
\hline 1 & $48(37.8 \%)$ & $48(38.7 \%)$ & $96(38.2 \%)$ \\
\hline 2 & $0(0.0 \%)$ & $1(0.8 \%)$ & $1(0.4 \%)$ \\
\hline \multicolumn{4}{|l|}{ Bulky disease } \\
\hline Yes & $58(45.7 \%)$ & $52(41.9 \%)$ & $110(43.8 \%)$ \\
\hline \multicolumn{4}{|l|}{ Extranodal lesions } \\
\hline Yes & $48(37.8 \%)$ & $53(42.7 \%)$ & $101(40.2 \%)$ \\
\hline \multicolumn{4}{|l|}{ IPI score } \\
\hline 0 & $39(30.7 \%)$ & $43(34.7 \%)$ & $82(32.7 \%)$ \\
\hline 1 & $85(66.9 \%)$ & $75(60.5 \%)$ & $160(63.7 \%)$ \\
\hline 2 & $3(2.4 \%)$ & $6(4.8 \%)$ & $9(3.6 \%)$ \\
\hline
\end{tabular}

All continuous values are reported with mean and coefficient of variation, while categories are reported in absolute numbers and percentages 
The time course of RXTM83 and rituximab clearly displayed bi-exponential declining serum concentration-time profiles and the fit of the two-compartment model to the data was markedly better than the one compartment model drug disposition in terms of objective function and diagnostic plots. Incorporation of the interoccasion variability (cycle 1 vs further cycles) also improved the fit significantly $(\triangle \mathrm{FMO}=-488, p<0.001)$. The results from the exploratory graphical and statistical analysis between the random effect of model parameters and the covariates evaluated revealed a significant trend of BSA with $V_{1}$ and in less extend with CL, and these covariates were formally tested in NONMEM to be incorporated into the model. However, the contribution of the effect of BSA on CL was not finally included as the contribution to the effect was limited with an exponent estimated to be 1 . The inclusion of BSA effect on $V_{1}$ reduced the IIV from 39 to $14 \%$.

The observed vs predicted plots stratified by arm show random normal scatter around the identity line indicating the absence of systematic bias and the adequacy of the model to describe the data. In addition, conditional weighted residuals also show random normal scatter around zero with no specific pattern suggesting model misspecification (Fig. 1).
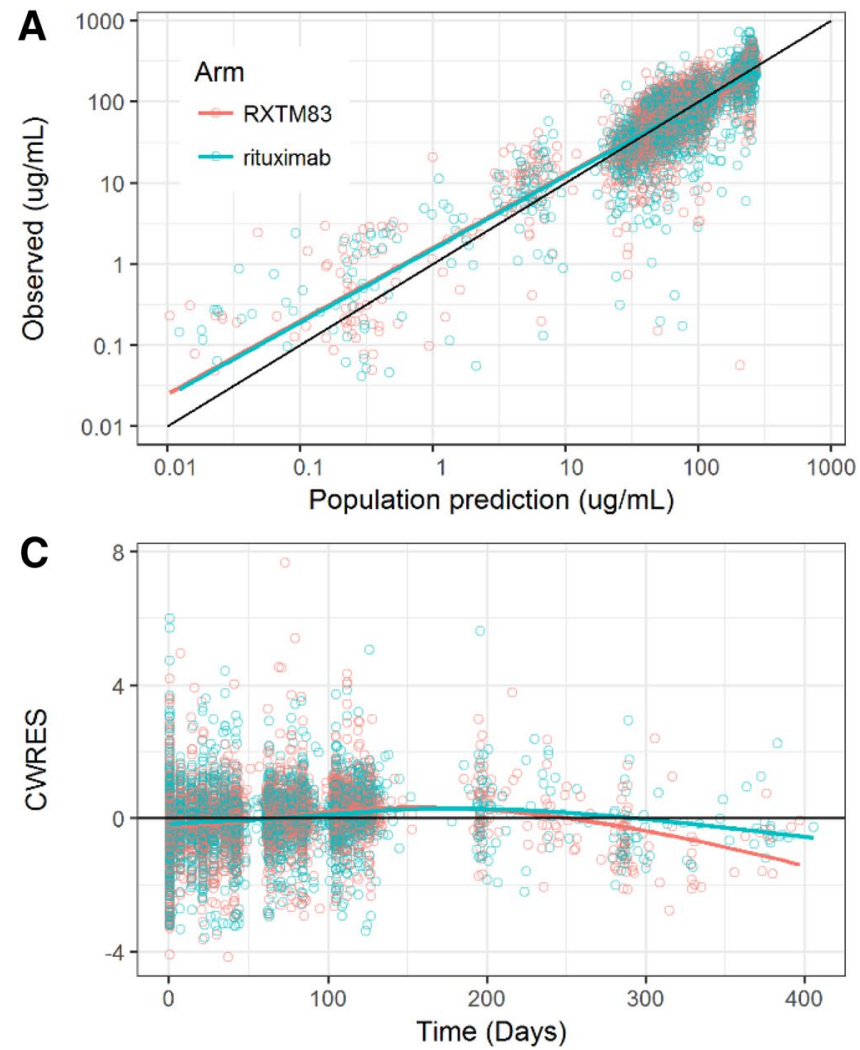

The estimates of the final model parameters together with the results of the non-parametric bootstrap are summarized in Table 2. IIV was estimated for all the model parameters and IOV was estimated for CL and $V_{1}$. Fixed parameters estimates were estimated with good precision, with all the relative standard error (RSE) less than 10\%; therefore, estimated $90 \%$ CI were narrow for all parameters. Moreover, IIV were lower than $30 \%$ and in agreement with values previously reported for rituximab [10, 26, 27]. IOV was higher for CL than for $V_{1}$ and both were well estimated with RSE lower than $27 \%$. In addition, the shrinkage was lower than $30 \%$ for all the parameters except for Q that was determined to be closed to $50 \%$, indicating the absence of model misspecification.

\section{Model qualification}

Results of the model qualification demonstrated the suitability of the developed model in the analysis population. The bootstrap distribution was compared to the point estimates obtained from the original data set (Table 2). Overall, the population estimates for the final model were very similar to the mean of the 1000 bootstrap replicates, and were contained within the $90 \%$ CIs obtained from the
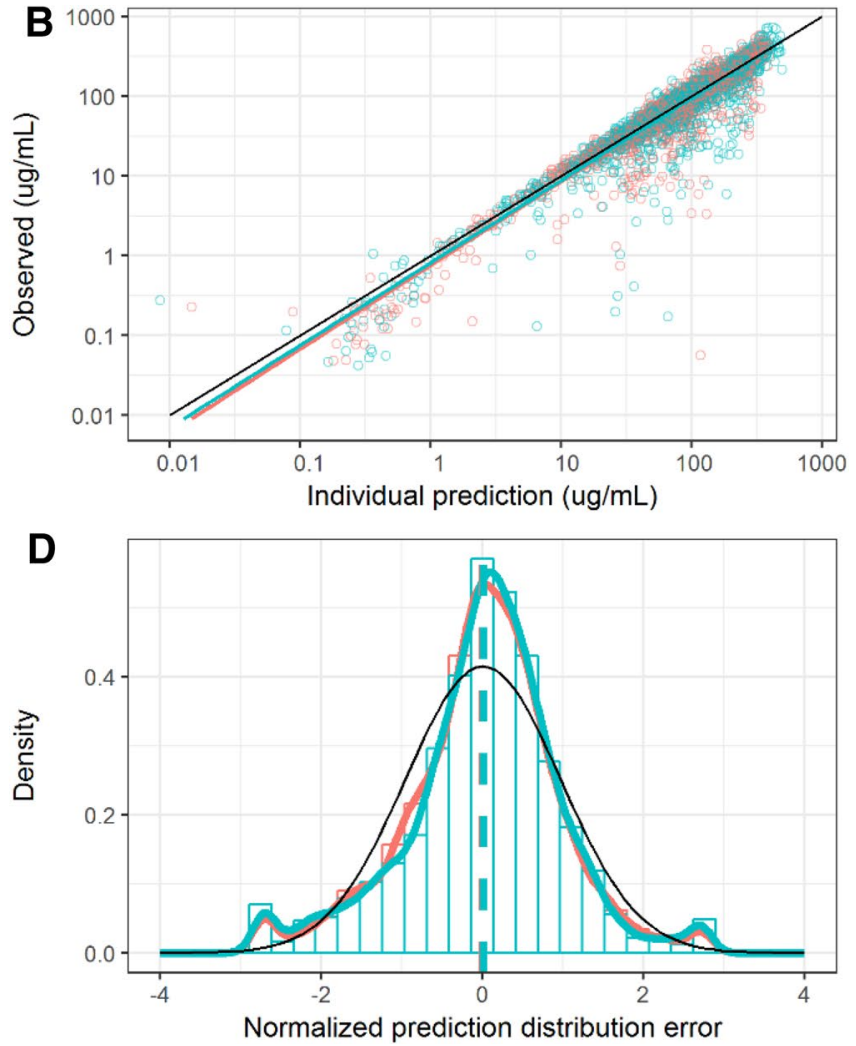

Fig. 1 Diagnostic plots for the final rituximab pharmacokinetic model. Observed vs predicted plots (a, b), conditional weighted residuals (c), and distribution error (d) stratified by treatment arm 
Table 2 Parameter estimates of the final population pharmacokinetic model for rituximab and bootstrap results

\begin{tabular}{|c|c|c|c|c|c|}
\hline \multirow[t]{2}{*}{ Parameter } & \multirow[t]{2}{*}{ Estimate (RSE, \%) } & \multirow[t]{2}{*}{$90 \% \mathrm{CI}^{\mathrm{a}}$} & \multicolumn{2}{|l|}{ Bootstrap } & \multirow[t]{2}{*}{ Shrinkage (\%) } \\
\hline & & & Median (RSE, \%) & $90 \% \mathrm{CI}$ & \\
\hline \multicolumn{6}{|c|}{ Fixed-effect parameters } \\
\hline $\mathrm{CL}(\mathrm{mL} / \mathrm{h})$ & $12.5(2.35)$ & $12.1-13.0$ & $12.5(2.40)$ & $12.0-13$ & - \\
\hline$V_{1}(\mathrm{~mL})$ & $3191(1.36)$ & $3120-3262$ & $3189(1.39)$ & $3115-3263$ & - \\
\hline$Q(\mathrm{~mL} / \mathrm{h})$ & $18.6(6.40)$ & $16.6-20.6$ & $18.6(4.74)$ & $17.2-20$ & - \\
\hline$V_{2}(\mathrm{~mL})$ & $4154(2.77)$ & $3965-4343$ & $4148(2.67)$ & $3973-4326$ & - \\
\hline$V_{1}$-BSA & $1.11(9.91)$ & $0.93-1.29$ & $1.11(9.53)$ & $0.941-1.3$ & - \\
\hline \multicolumn{6}{|c|}{ Random-effect parameters (CV, \%) } \\
\hline $\mathrm{IIV}_{\mathrm{CL}}^{\mathrm{b}}$ & $24.7(26.4)$ & $18.4-29.8$ & $24.2(27.8)$ & $18.3-29.7$ & 33.6 \\
\hline $\mathrm{IIV}_{V 1 \mathrm{~b}}$ & $14.2(25.0)$ & $10.9-16.9$ & $14.0(25.4)$ & $10.7-16.9$ & 35.7 \\
\hline $\mathrm{IIV}_{Q}^{\mathrm{b}}$ & $28.0(72.6)$ & $0.00-42.5$ & $27.1(70.1)$ & $0.01-40.7$ & 48.8 \\
\hline $\mathrm{IIV}_{V 2}{ }^{\mathrm{b}}$ & $27.0(17.5)$ & $22.7-30.8$ & $27.1(17.4)$ & $23-31$ & 29.4 \\
\hline $\mathrm{IOV}_{\mathrm{CL}}^{\mathrm{b}}$ & $35.9(11.9)$ & $32.0-39.5$ & $35.8(12.2)$ & $31.9-39.8$ & 21.8 \\
\hline $\mathrm{IOV}_{V 1}^{\mathrm{b}}$ & $16.8(26.4)$ & $12.6-20.2$ & $16.6(28.4)$ & $12.9-20.5$ & 26.4 \\
\hline \multicolumn{6}{|l|}{ Residual variability } \\
\hline Proportional (\%) & $27(3.29)$ & $25.5-28.4$ & $26.9(3.33)$ & $25.4-28.5$ & 7.83 \\
\hline Additive (ng/mL) & $278(26.5)$ & $157-399$ & $279(21.2)$ & $198-395$ & - \\
\hline
\end{tabular}

$I I V$ interindividual variability, $I O V$ interoccasion variability, $C V$ coefficient of variation, $C I$ confidence interval, $R S E$ relative standard error, $C L$ systemic clearance, $V_{l}$ central volume of distribution, $Q$ intercompartmental clearance, $V_{2}$ peripheral volume of distribution

${ }^{a}$ Asymptotic CI derived from NONMEM standard errors

${ }^{\mathrm{b}} \mathrm{CV}=100 \times \operatorname{sqrt}(\exp ($ variance $)-1)$

${ }^{\mathrm{c}}$ Epsilon shrinkage is the overall shrinkage for related elements of the residual error bootstrap analysis. This suggested the precision of the NONMEM parameter estimates and the absence of bias. The results of the VPC evidence that the model developed is appropriate to describe the time course of RXTM83 and rituximab and their variability in DLBCL patients. The overall distribution of the NPDE is presented in Fig. 1d and approximately follows a normal distribution with a mean of 0 and a standard deviation (SD) of 1 (Fig. 1d).

\section{Pharmacokinetic assessment}

Statistical analysis of primary PK endpoints by treatment cycle and arm was assessed for PK similarity (Table 3). The ratios $(90 \% \mathrm{CI})$ of geometric least-square means (RXTM83 to rituximab treatment group) were $0.992(0.936-1.05)$ for AUC and $0.996(0.939-1.05)$ for $C_{\max }$. Similar results of RXTM83 were obtained when the PK of rituximab at steady state (cycle 6) was evaluated. In this case, the corresponding values were $1.03(0.985-1.07)$ for AUC and 1.04 (0.995-1.09) for $C_{\max }$. Furthermore, the comparison
Table 3 Statistical analysis of pharmacokinetic primary endpoints for cycle 1 and cycle 6 (steady state)

\begin{tabular}{|c|c|c|c|c|c|c|}
\hline Parameter (unit) & Occasion & Treatment & $N$ & $\begin{array}{l}\text { Geometric } \\
\text { of LS means }\end{array}$ & $\begin{array}{l}\text { Ratio of } \\
\text { geometric LS } \\
\text { means }\end{array}$ & $90 \% \mathrm{CI}$ of the ratio \\
\hline \multirow[t]{4}{*}{$\mathrm{AUC}_{0-\infty}(\mathrm{h} \mu \mathrm{g} / \mathrm{mL})$} & Cycle 1 & RXTM83 & 127 & 44,519 & 0.992 & $0.936-1.05$ \\
\hline & & Rituximab & 123 & 44,874 & & \\
\hline & Cycle 6 & RXTM83 & 113 & 60,875 & 1.03 & $0.985-1.07$ \\
\hline & & Rituximab & 105 & 59,079 & & \\
\hline \multirow[t]{4}{*}{$C_{\max }(\mu \mathrm{g} / \mathrm{mL})$} & Cycle 1 & RXTM83 & 127 & 196.8 & 0.996 & $0.939-1.05$ \\
\hline & & Rituximab & 123 & 197.5 & & \\
\hline & Cycle 6 & RXTM83 & 113 & 291 & 1.04 & $0.995-1.09$ \\
\hline & & Rituximab & 105 & 279 & & \\
\hline
\end{tabular}

$N$ number of patients, $L S$ least squares, $C I$ confidence interval 
analysis of the individual PK parameters derived from the developed model showed that RTXM83 PK and its associated variability were similar to the reference product, with geometric mean (CV\%) of CL and $V_{1}$ of $14.4(42 \%)$ vs 14.3 (44\%) $\mathrm{mL} / \mathrm{h}$ and 3.18 (23\%) vs 4.1 (20\%) L for RXTM83 and rituximab reference product, respectively, after single infusion of rituximab. Results after repeated infusions also showed the PK similarity between RXTM83 and rituximab reference product with geometric mean (CV\%) of CL and $V_{1}$ of $10.6(33 \%)$ vs $11.1(33 \%) \mathrm{mL} / \mathrm{h}$ and $3.13(19 \%)$ vs 4.11 (21\%) L, respectively (Table 4).

\section{Pharmacodynamics}

Immediately after the first administration of RTXM83 or the reference product (co-administered with CHOP), the median percent changes from baseline of both $\mathrm{CD} 20+$ and CD19+ B-cell suffered a remarkable change. At the first EOI, CD20+ B-cells counts reached $100 \%$ depletion and in the same magnitude in both treatment arms (median change from baseline of 100\%). In the same manner, CD19+ B-cells almost achieved complete depletion ( $>99 \%$ ) around day 7 (approximately $168 \mathrm{~h}$ hours) after the first treatment infusion of RTXM83 or the reference product (99.8 and 99.6\%, respectively). A complete depletion of CD20+ and CD19+ B-cells was observed during the following treatment cycles. A modest recovery of B-cells started around 6th month after last rituximab treatment administration, with a $60 \%$ of patients having achieved a significant B-cell recovery by the 9th month after treatment. B-cell depletion, length of suppression, or time to recovery to normal B-cell showed to be similar between both treatment arms (Fig. 2).

\section{Immunogenicity}

Overall ADA incidence in the studied population was low and similar between RTXM83 and the rituximab reference product. The proportion of patients who developed ADA de novo (seroconversion) following rituximab administration was $\leq 4 \%$ in both arms. Treatment-induced ADA-positive samples were detected mainly during the follow-up observation period.

Among the patients who developed ADA de novo, no impact on their PK/PD profile was detected. Clearance median value was similar between ADA-positive subject and ADA-negative subject in both RTXM83 (10.8 vs $11.3 \mathrm{~mL} /$ min, respectively) and rituximab reference product (13.6 vs $11.3 \mathrm{~mL} / \mathrm{min}$, respectively). Regarding PD data, those ADApositive subjects with $\mathrm{PD}$ sample available did not show any significant difference in the depletion of CD20+ B-cells.

\section{Discussion}

To our knowledge, this is the first study to investigate the population PK of a rituximab biosimilar with a large sample size of patients, serum concentration, and cycles of treatment. A total of 251 DLBCL patients from 58 research sites in 12 countries participating in the study were included in the study and 5341 serum concentrations with 1512 cycles of treatment in total (776 and 736 for RXTM83-CHOP and $\mathrm{R}-\mathrm{CHOP}$, respectively) were included in the analysis data set.

The developed population PK model for rituximab allowed the use of the sparse sampling and the quantification of any potential differences in PK parameters between
Table 4 Summary of individual pharmacokinetic parameters estimates by treatment arm at cycle 1 and at cycle 6 (at steady state)

\begin{tabular}{llll}
\hline & & RTXM83-CHOP & Rituximab-CHOP \\
\hline Cycle 1 & & & \\
Elimination clearance (mL/h) & Geometric mean (CV\%) & $14.43(41.7 \%)$ & $14.29(43.5 \%)$ \\
& Range & $4.93-34.25$ & $4.12-48.90$ \\
Volume of distribution, central $(\mathrm{mL})$ & Geometric mean (CV\%) & $3181.40(22.8 \%)$ & $3138.10(27.4 \%)$ \\
& Range & $1361.51-5174.53$ & $1288.91-6218.79$ \\
Distribution clearance (mL/h) & Geometric mean (CV\%) & $18.23(14.9 \%)$ & $18.27(13.4 \%)$ \\
& Range & $9.26-23.95$ & $11.69-27.12$ \\
Volume of distribution, peripheral (mL) & Geometric mean (CV\%) & $4106.39(20.0 \%)$ & $4136.75(17.7 \%)$ \\
& Range & $2505.55-7602.77$ & $2662.05-7634.76$ \\
Cycle 6 at steady state & & & \\
Clearance (mL/h) & Geometric mean (CV\%) & $10.6(31.1 \%)$ & $11.1(33.0 \%)$ \\
& & $5.0-28.2$ & $4.7-26.0$ \\
Volume of distribution, central (mL) & Geometric mean (CV\%) & $3129.7(18.6 \%)$ & $3166.5(22.9 \%)$ \\
& Range & $1950.7-5150.3$ & $1576.9-5691.5$ \\
Distribution clearance (mL/h) & Geometric mean (CV\%) & $18.3(15.3 \%)$ & $18.5(12.6 \%)$ \\
& Range & $9.3-23.9$ & $11.7-27.1$ \\
Volume of distribution, peripheral (mL) & Geometric mean (CV\%) & $4113.2(20.9 \%)$ & $4148.6(18.5 \%)$ \\
& Range & $2505.6-7602.8$ & $2662.1-7634.8$ \\
\hline
\end{tabular}


Fig. 2 Median percent changes (\%) from baseline in blood of CD20 (a) or CD19 (b) following intravenous administration of $375 \mathrm{mg} / \mathrm{m}^{2} \mathrm{q} 3 \mathrm{w}$ RTXM 83 or rituximab reference product co-administered with CHOP in cycles 1-6 (including follow-up period 3)
A

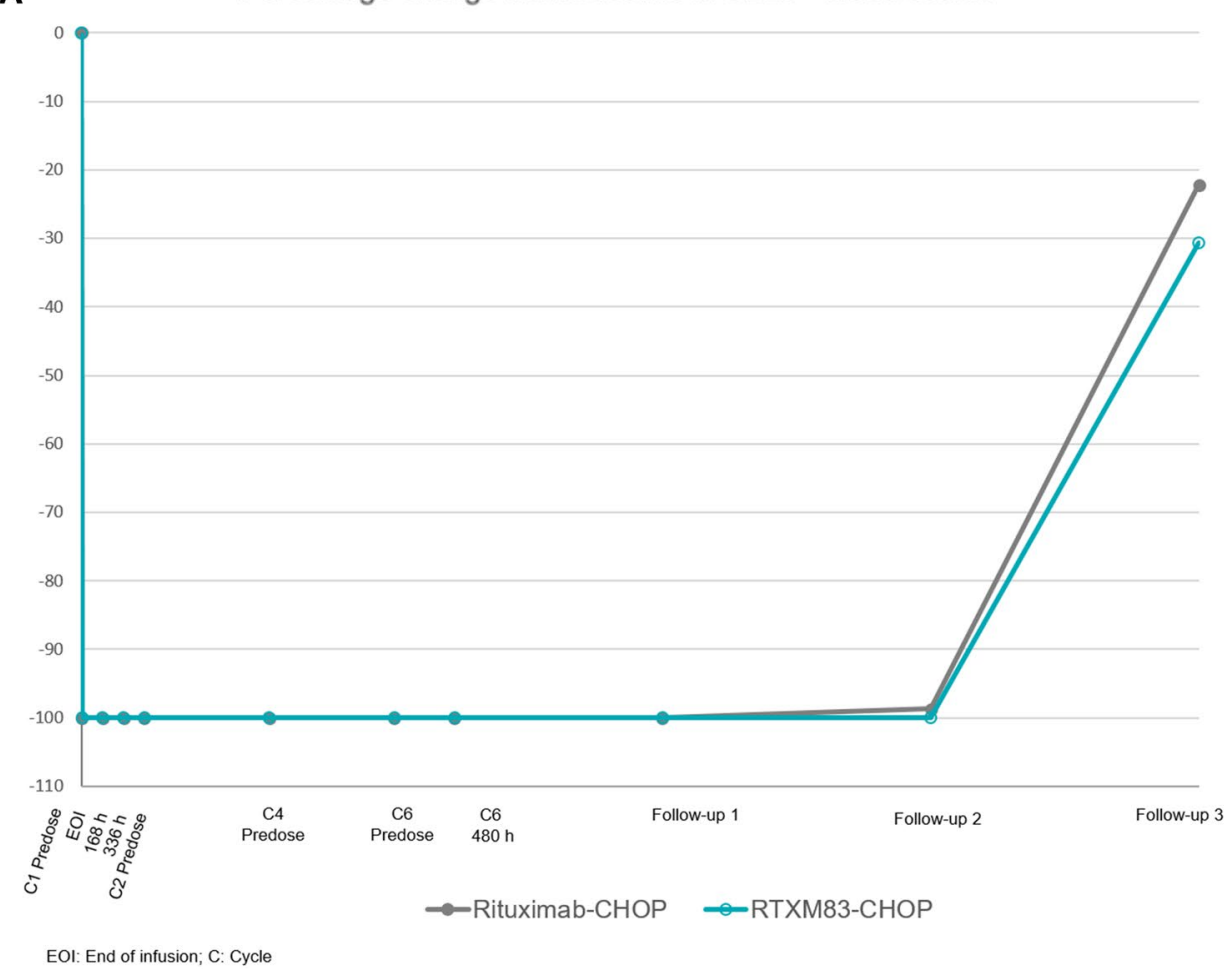

B Percentage change from baseline in CD19+ blood counts

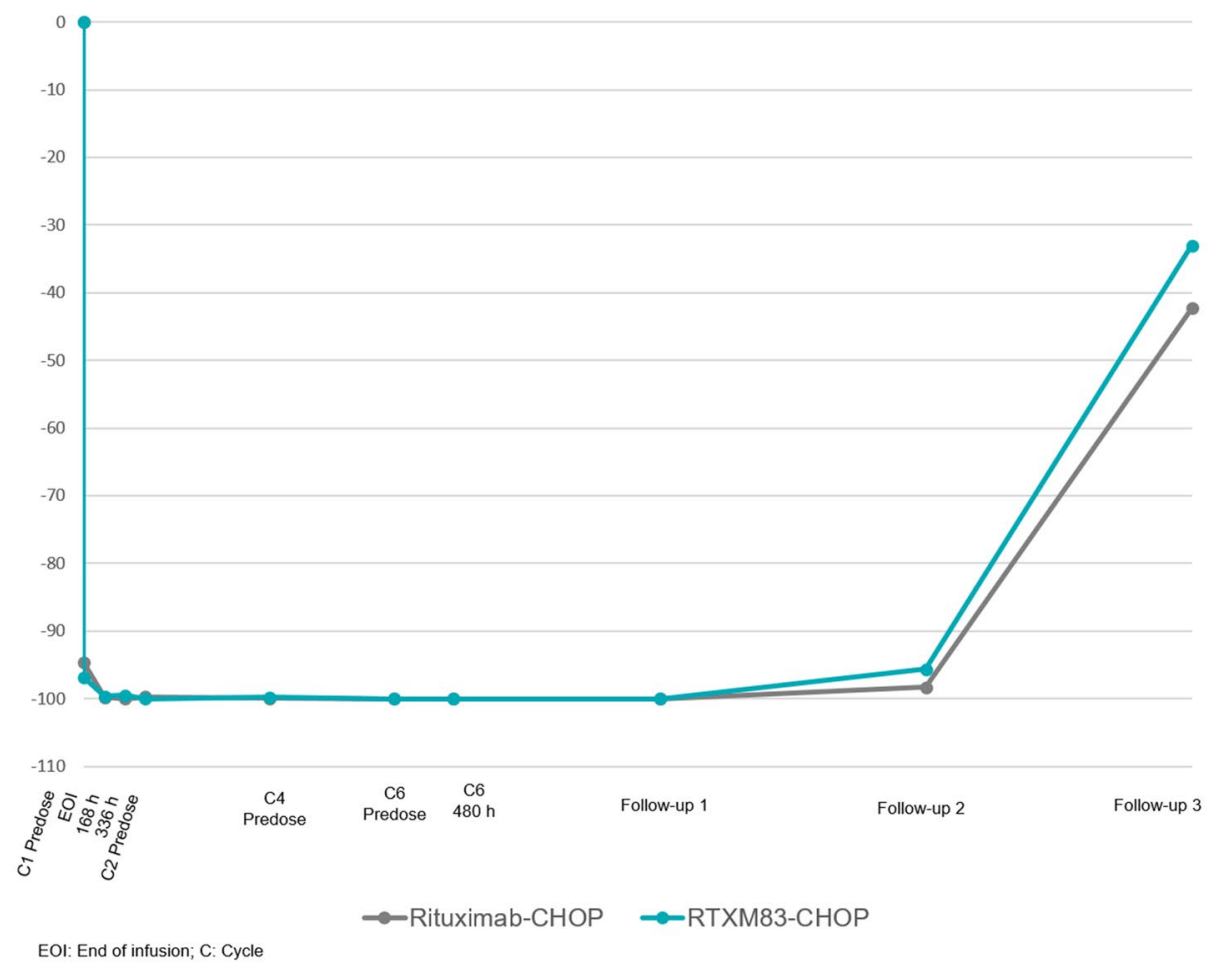

the treatments. Population PK approach has been used previously with rituximab in DLBCL patient, either as the reference product [12, 13, 22, 23] or with a rituximab biosimilar
[23], but these studies had a different study design, sample size, scheme of treatment, and patient characteristics [22]. For instance, the study done by Muller et al. included a 
smaller sample size (less than 20 patients) and the median age of patients analysed in that study was higher (69 years old) than in our study (51 years old) [13]. The larger PK population analysed in our study (251 patients) increased the power to detect potential differences between the biosimilar candidate and the originator.

The use of a model-based analysis with sparse data taken at fixed time intervals to investigate bioequivalence might be considered a major limitation of our study. However, non-compartmental approach requires a rich sampling profile to calculate AUC by a trapezoidal rule, and the choice of sampling times can strongly influence the assessment of $C_{\max }$. By comparison, a model-based analysis can require many fewer samples per subject with the expectation of similar sensitivity to differences in PK between originator and biosimilar allowing the PK comparison in the clinical setting. Actually, rationally informative samplings have been selected in our study based on a PK model for the originator, prior information on the $\mathrm{PK}$ properties of the originator, and the desired patient trial design, all of which counteracts the possible limitation of the sparse sampling.

Other rituximab biosimilars have demonstrated bioequivalence in population of patients with rheumatoid arthritis at week 24 after a single dose, or after two doses administrated on day 1 and day 15 [24, 25]. In contrast, in this study, rituximab PK was assessed in DLBCL population, not only after single dose but also after multiple doses to achieve steady-state concentrations following the recommendations of FDA guidelines on bioequivalence $[20,26]$. This population has allowed us to have a full characterization of the PK profile and a complete comparison between the biosimilar and the reference medicinal product PK.

Covariates and potential factors that could alter the PK of rituximab were well balanced between the two treatment arms, indicating that the presence of bias in the analysed PK data set was unlikely. Noteworthy, potential known factors that could alter the PK of rituximab such as body weight (median values of 66 and $65.8 \mathrm{~kg}$ for RXTM83 and rituximab, respectively), BSA (1.73 vs $1.71 \mathrm{~m}^{2}$ ), and tumour burden ( 89.1 vs 63.3 cells $/ \mu \mathrm{L}$ ) were balanced between treatment arms.

In addition, the assessment of other factors such as induced ADA response that could affect the PK profile, has shown in these results no impact in any of the study arms or any relationship with their PK/PD profile.

Population PK parameters estimated from RXTM83AC-01-11 were in line with that reported for rituximab when used in different indications [13, 14, 27, 28]. The evaluated covariates, which included demographics and disease-related factors, had no significant effect on the PK of rituximab except for BSA that was included in the model and explained about $5 \%$ of the interindividual variance for
$V_{1}$. This effect has been previously described for rituximab [27]. No influence of tumour burden was observed in the study, though the influence of tumour burden is known to alter the PK of rituximab. The retention of rituximab by its tumour target antigen is associated with decreased serum concentrations and, consequently, with increased volumes of distribution and elimination half-live. This discrepancy regarding tumour burden has been noted in other studies $[12,29]$ who found the level of rituximab exposure to be similar after several infusions. If tumour burden is inversely correlated with rituximab, serum concentration is still a question of debate [30]. This phenomenon may also explain the differences observed for other studies [31]. In addition, reported values of rituximab PK in different diseases as follicular lymphoma and chronic lymphocytic leukemia showed similar results [14, 28].

Because of the high degree of similarity between the PK parameters derived from the population model, the results of the analysis evidenced that RTXM83 was similar to the rituximab reference product with a proper analysis. The concentration vs time profiles and all PK parameters indicated close similarity of RTXM83 and the reference product across repeated cycles of treatment, and it can be concluded that bioequivalence was formally demonstrated.

The ratios $(90 \% \mathrm{CI})$ of geometric least-square means (RXTM83 to rituximab treatment group) met the expectation for statistical equivalence for $\mathrm{AUC}$ and for $C_{\max }$ at cycle 1 and at steady state. Therefore, the systemic exposure (AUC and $C_{\max }$ ) and its associated variability derived from the developed PK model for rituximab demonstrated that the PK of RTXM83 and the reference product were similar.

B-cell counts is a useful parameter to assess rituximab activity and was assessed as a secondary endpoint in the study. Typically, B-cell count falls to barely detectable level after administration of rituximab and then gradually recovers to baseline after 6 months following the last dose [32]. The patients included in our study showed a pattern of CD20+ and $\mathrm{CD} 20+\mathrm{B}$-cell depletion and recovery comparable between RTXM83 and the rituximab reference product.

\section{Conclusion}

To our knowledge, this is the first study to provide a description of the PK of the rituximab biosimilar RTXM83 in a large population and to provide a well-characterized, robust PK method that measures the biosimilar and reference products with comparable precision and accuracy. The time course of RTXM83 was well characterized by the model developed. The use of a non-linear mixed effect modelling population approach when assessing clinical comparability exercise of RTXM83 to rituximab reference product 
facilitated the inclusion of sparse data improving the power to detect any potential differences between RTXM83 and rituximab reference product. This analysis demonstrated that the systemic exposure (AUC and $C_{\max }$ ) of RTXM83 and its associated variability were similar to those for rituximab in DLBCL patients. Moreover, the profile in CD20+ and CD20+ B-cell depletion also was similar between RTXM83 and Mabthera ${ }^{\circledR}$ and consistent with the Mabthera ${ }^{\circledR}$ bibliography, supporting its claimed biosimilarity with the reference product.

Acknowledgements The authors would like to acknowledge Belén Valenzuela and Daniel Röshammar of the SGS Exprimo NV company for their support with this article. Other institutions and principal investigators who participated in the study are as follows: Dr. Surender Kumar Beniwal, Acharya Tulsi Regional Cancer Treatment and Research Institute (RCC), S. P. Medical College and AG of Hospitals, Bikaner, Rajasthan, India. Dr. Lokanath D HCG Care Centre, Bangalore, India. Dr. Niraj Bhatt and Dr. Pramod Patil, Kailash Cancer Hospital and Research Centre, Vadodara, India. Dr. Daniel Oscar Bär, Hospital J.B. Iturraspe, Santa Fe, Santa Fe, Argentina. Dr. Asis Mukhopadhyay, Netaji Subhash Chandra Bose Cancer Research Institute, Kolkata, India. Dr. Marcia Torresan Delamain, Universidade Estadual de Campinas (UNICAMP), Campinas, SP, Brazil. Dr. David Hugo Flores, Hospital Ángel Padilla, San Miguel de Tucumán, Tucumán,Argentina. Dr. Shibashish Bhattacharyya, Health Point Hospital, Kolkata, India. Dr. John Querol, Veterans Memorial Medical Center (VMMC), Diliman, Quezon City, Philippines. Dr. Alejandro Salvatierra, Consultorios Médicos de la Fundación ARS Médica, San Salvador de Jujuy, Jujuy, Argentina. Dr. Dinesh Bhurani, Rajiv Gandhi Cancer Institute and Research Centre, Delhi, India. Dr. Sandeep Jasuja, Birla Cancer CenterSMS Hospital, Jaipur, India. Dr. Venkatraman Radhakrishnan, Cancer Institute, Chennai, India. Dr. Syafrizal Syafei, Dharmais National Cancer Center, Jakarta, Indonesia. Dr. Rubén Dario Kowalyszyn, Centro de Investigaciones Clínica-Clínica Viedma SA, Viedma, Rio Negro, Argentina. Prof Moosa Patel, Chris Hani Baragwanath Hospital, Soweto, South Africa. Dr. Sérgio Shusterschitz da Silva Araújo Hospital das Clínicas-UFMG, Belo Horizonte, MG, Brazil. Dr. Lipatov Oleg Nikolaevich, State Budgetary Healthcare Institution "Republican Clinical Oncology Dispensary", Ufa, Russian Federation. Dr. Volodicheva Elena Mikhailovna, State Healthcare Institution of Tula region "Tula regional clinical hospital", Tula, Russian Federation. Dr. Sayyed Reza Safaei Nodehi, Imam Khomeini Complex Hospital, Teheran, Iran. Dr. Toh See Guan, Hospital Sultanah Aminah, Johor, Malaysia. Dr. Julia Caffaro and Dr. Giselda De Stefano, Instituto Roffo, Capital Federal, Buenos Aires, Argentina. Dr. Felycette Gay P. MartinezLapus, Davao Doctors Hospital-DDH, Davao, Philippines. Dr. Ellie May Villegas, Perpetual Succour Hospital-PSH, Cebu, Philippines. Dr. Juan Jose García, Hospital Privado Centro Médico de Córdoba, Córdoba, Córdoba, Argentina. Dr. Carlos Bermudez and Dr Alberto M. Pereira, Instituto Nacional de Cancerología, Bogota, Colombia. Dr. Rakesh Raman, Mount Miriam Cancer Hospital, Penang, Malaysia. Dr. Prof. Gan Gin, University Malaya Medical Centre-UMMC, Kuala Lumpur, Malaysia. Dr. Laura Fogliatto, Santa Casa de Porto Alegre, Porto Alegre, RS, Brazil. Dr. Eduardo Cilião Munhoz, Hospital Erasto Gaertner-Paranaense de Combate ao Câncer, Curitiba, PR, Brazil. Dr. Gonzalo Ramiro Molina Barrios, Centro Oncológico Riojano Integral (CORI), La Rioja, La Rioja, Argentina. Dr. Proydakov Andrey Viktorovich, State Institution "Komi Republican Oncology Dispensary", Syktyvkar, Russian Federation. Dr. Beatrice J. Tiangco, National Kidney and Transplant Institute (NKTI), Manila, Philippines. Dr. Manikhas Georgy Moiseevich, Saint-Petersburg State Budgetary Institution "City Clinical Oncology Dispensary”, Saint-Petersburg, Russian Federation.
Dr. Martin Miguel Saslavsky, Instituto de Hematología y Medicina Clínica "Dr. Rubén Dávoli”, Rosario, Santa Fe, Argentina. Dr. Graciela Inés Avila, Hospital Gral. De Agudos Donación Francisco Santojanni, Liniers, Buenos Aires, Argentina. Dr Leaanne Jones and Dr Jorn Malan, GVI Oncology, Port Elizabeth, South Africa. Dr. Guillermo Zenón Beguelin, Clínica Radiológica del Sur, Cipolletti, Argentina. Dr. Noemi A. Uy, Cebu Doctors University Hospital-CDUH, Cebu, Philippines. Dr. Joaquin D. Rosales, Fundación Valled de Lili, Cali, Valle del Cauca, Colombia. Dr. Miguel Muñoz, Centro Oncológico de Rosario, Rosario, Santa Fe, Argentina. Dr. Marco Aurélio Salvino de Araujo, Monte Tabor-Hospital São Rafael, Salvador, BA, Brazil. Dr Fatima Bassa, Tygerberg Academic Hospital, Cape Town, South Africa. Dr Kenny Galvez, Hospital Pablo Tobón Uribe, Medellín, Antioquia, Colombia. Dr. Juliana Pereira, Hospital das Clínicas-FMUSP, São Paulo, SP, Brazil. Dr. Roberto Luiz da Silva, Centro de Pesquisa do Instituto Brasileiro de Controle do Câncer-IBCC, São Paulo, SP, Brazil. Dra. Patrícia Xavier Santi, CEPHO-Centro de Estudos e Pesquisas de Hematologia e Oncologia, Faculdade de Medicina do ABC, Santo André, SP, Brazil. Dr. José Salvador Rodrigues de Oliveira Hospital Santa Marcelina, São Paulo, SP, Brazil. Dr. Márcio Debiasi, Hospital São Lucas da PUCRS, Porto Alegre, RS, Brazil. Dr. Éderson Roberto de Mattos, Hospital Amaral Carvalho, Jaú, SP, Brazil. Dr. Laura Fogliatto, Hospital das Clínicas de Porto Alegre-Centro de Pesquisa Clínica, Porto Alegre, RS, Brazil. Dr. Nicolas Silva Lazaretti, Hospital da Cidade, Passo Fundo, RS, Brazil. Dr. Burdaeva Olga Nikolaevna, State Budgetary Healthcare Institution of Arkhangelsk region "Arkhangelsk Clinical Oncology Dispensary, Arkhangelsk, Russian Federation. Dr. Melnichenko Vladimir Yaroslavovich, National Medical Surgical Center n.a. N.I. Pirogov, Moscow, Russian Federation. Dr. Chew Teng Keat, Penang General Hospital, Pulau Pinang, Malaysia. Dr. Osmanov Dzhelil Shevketovich, Russian Oncology Scientific Center n.a. N.N. Blokhin, Moscow, Russian Federation.

Funding This study was supported by Mabxience Research SL.

\section{Compliance with ethical standards}

Conflict of interest S. Millan, A. Paravisini, L. Perez, FJ. Fernández, and (A) Del Campo Garcia are employees of Mabxience Research SL. (B) Miguel-Lillo served in a consultant/advisory role to Mabxience Research SL. M Candelaria and D. Gonzalez have received a research funding from Mabxience Research SL.

Ethical approval All procedures performed in studies involving human participants were in accordance with the ethical standards of the institutional and/or national research committee and with the 1964 Helsinki declaration and its later amendments or comparable ethical standards.

Informed consent Informed consent was obtained from all individual participants included in the study.

Open Access This article is distributed under the terms of the Creative Commons Attribution 4.0 International License (http://creativecommons.org/licenses/by/4.0/), which permits unrestricted use, distribution, and reproduction in any medium, provided you give appropriate credit to the original author(s) and the source, provide a link to the Creative Commons license, and indicate if changes were made. 


\section{References}

1. Coleman M, Lammers PE, Ciceri F, Jacobs IA (2016) Role of rituximab and rituximab biosimilars in diffuse large B-cell lymphoma. Clin Lymphoma Myeloma Leuk 16(4):175-181. https:// doi.org/10.1016/j.clml.2016.01.004

2. EMA (2014) Guideline on similar biological medicinal products (CHMP/437/04 Rev 1). http://www.ema.europa.eu/docs/en_GB/ document_library/Scientific_guideline/2014/10/WC500176768. pdf

3. FDA (2016) Guidance for industry. Clinical pharmacology data to support a demonstration of biosimilarity to a reference product. http://www.fda.gov/downloads/drugs/guidancecomplianceregulatoryinformation/guidances/ucm397017.pdf

4. FDA (1999) Guidance for industry. Population pharmacokinetics. https://www.fda.gov/downloads/Drugs/GuidanceComplianceRegulatoryInformation/Guidances/UCM072137.pdf

5. Cuello HA, Segatori VI, Alberto M, Pesce A, Alonso DF, Gabri MR (2016) Comparability of antibody-mediated cell killing activity between a proposed biosimilar RTXM83 and the originator rituximab. BioDrugs 30(3):225-231. https://doi.org/10.1007/ s40259-016-0171-8

6. Coiffier B, Lepage E, Briere J, Herbrecht R, Tilly H, Bouabdallah R, Morel P, Van Den Neste E, Salles G, Gaulard P, Reyes F, Lederlin P, Gisselbrecht C (2002) CHOP chemotherapy plus rituximab compared with $\mathrm{CHOP}$ alone in elderly patients with diffuse large-B-cell lymphoma. N Engl J Med 346(4):235-242. https://doi.org/10.1056/NEJMoa011795

7. Pescovitz MD (2006) Rituximab, an anti-cd20 monoclonal antibody: history and mechanism of action. Am J Transpl 6(5 Pt 1):859-866. https://doi.org/10.1111/j.1600-6143.2006.01288.x

8. Shankar G, Devanarayan V, Amaravadi L, Barrett YC, Bowsher R, Finco-Kent D, Fiscella M, Gorovits B, Kirschner S, Moxness M, Parish T, Quarmby V, Smith H, Smith W, Zuckerman LA, Koren E (2008) Recommendations for the validation of immunoassays used for detection of host antibodies against biotechnology products. J Pharm Biomed Anal 48(5):1267-1281. https://doi. org/10.1016/j.jpba.2008.09.020

9. Beal SL, Boeckmann AJ, Bauer RJ (eds) (1989-2011) NONMEM users guides. Icon development solutions, Ellicott City, Maryland, USA

10. Lindbom L, Pihlgren P, Jonsson EN (2005) PsN-Toolkit-a collection of computer intensive statistical methods for non-linear mixed effect modeling using NONMEM. Comput Methods Programs Biomed 79(3):241-257. https://doi.org/10.1016/j. cmpb.2005.04.005

11. Blasco H, Chatelut E, de Bretagne IB, Congy-Jolivet N, Le Guellec C (2009) Pharmacokinetics of rituximab associated with CHOP chemotherapy in B-cell non-Hodgkin lymphoma. Fundam Clin Pharmacol 23(5):601-608. https://doi. org/10.1111/j.1472-8206.2009.00714.x

12. Muller C, Murawski N, Wiesen MH, Held G, Poeschel V, Zeynalova S, Wenger M, Nickenig C, Peter N, Lengfelder E, Metzner B, Rixecker T, Zwick C, Pfreundschuh M, Reiser M (2012) The role of sex and weight on rituximab clearance and serum elimination half-life in elderly patients with DLBCL. Blood 119(14):32763284. https://doi.org/10.1182/blood-2011-09-380949

13. Regazzi MB, Iacona I, Avanzini MA, Arcaini L, Merlini G, Perfetti V, Zaja F, Montagna M, Morra E, Lazzarino M (2005) Pharmacokinetic behavior of rituximab: a study of different schedules of administration for heterogeneous clinical settings. Ther Drug Monit 27(6):785-792

14. Mandema JW, Verotta D, Sheiner LB (1992) Building population pharmacokinetic-pharmacodynamic models. I. Models for covariate effects. J Pharmacokinet Biopharm 20(5):511-528
15. Efron BTR (1993) An introduction to the bootstrap. Chapman and Hall/CRC Press., London

16. Brendel K, Comets E, Laffont C, Laveille C, Mentre F (2006) Metrics for external model evaluation with an application to the population pharmacokinetics of gliclazide. Pharm Res 23(9):20362049. https://doi.org/10.1007/s11095-006-9067-5

17. Nguyen TH, Comets E, Mentre F (2012) Extension of NPDE for evaluation of nonlinear mixed effect models in presence of data below the quantification limit with applications to HIV dynamic model. J Pharmacokinet Pharmacodyn 39(5):499-518. https://doi. org/10.1007/s10928-012-9264-2

18. Yano Y, Beal SL, Sheiner LB (2001) Evaluating pharmacokinetic/ pharmacodynamic models using the posterior predictive check. $\mathrm{J}$ Pharmacokinet Pharmacodyn 28(2):171-192

19. FDA (2014) Guidance for Industry. Bioavailability and Bioequivalence Studies Submitted in NDAs or INDs-General Consideration. http://www.fda.gov/downloads/Drugs/GuidanceComplianceRegulatoryInformation/Guidances/UCM389370.pdf

20. EMA (2010) Guideline on the investigation of bioequivalence (CHMP/1401/98). http://www.ema.europa.eu/docs/en_GB/document_library/Scientific_guideline/2010/01/WC500070039.pdf

21. Rozman S, Grabnar I, Novakovic S, Mrhar A, Jezersek Novakovic B (2017) Population pharmacokinetics of rituximab in patients with diffuse large B-cell lymphoma and association with clinical outcome. Br J Clin Pharmacol 83(8):1782-1790. https://doi. org/10.1111/bcp.13271

22. Gota V, Karanam A, Rath S, Yadav A, Tembhare P, Subramanian P, Sengar M, Nair R, Menon H (2016) Population pharmacokinetics of Reditux, a biosimilar Rituximab, in diffuse large B-cell lymphoma. Cancer Chemother Pharmacol 78(2):353-359. https:// doi.org/10.1007/s00280-016-3083-x

23. EMA (2016) Truxima-EPAR (EMA/CHMP/75695/2017). http:// www.ema.europa.eu/docs/en_GB/document_library/EPAR_-_ Public_assessment_report/human/004112/WC500222695.pdf

24. Smolen JS, Cohen SB, Tony HP, Scheinberg M, Kivitz A, Balanescu A, Gomez-Reino J, Cen L, Zhu P, Shisha T (2017) A randomised, double-blind trial to demonstrate bioequivalence of GP2013 and reference rituximab combined with methotrexate in patients with active rheumatoid arthritis. Ann Rheum Dis 76(9):1598-1602. https://doi.org/10.1136/ annrheumdis-2017-211281

25. EMA (2014) Guideline on similar biological medicinal products containing biotechnology-derived proteins as active substance: non-clinical and clinical issues (EMEA/CHMP/ BMWP/42832/2005 Rev1). http://www.ema.europa.eu/docs/ en_GB/document_library/Scientific_guideline/2015/01/ WC500180219.pdf

26. Ng CM, Bruno R, Combs D, Davies B (2005) Population pharmacokinetics of rituximab (anti-CD20 monoclonal antibody) in rheumatoid arthritis patients during a phase II clinical trial. J Clin Pharmacol 45(7):792-801. https://doi. org/10.1177/0091270005277075

27. Li J, Zhi J, Wenger M, Valente N, Dmoszynska A, Robak T, Mangat R, Joshi A, Visich J (2012) Population pharmacokinetics of rituximab in patients with chronic lymphocytic leukemia. J Clin Pharmacol 52(12):1918-1926. https://doi. org/10.1177/0091270011430506

28. Mangel J, Buckstein R, Imrie K, Spaner D, Franssen E, Pavlin P, Boudreau A, Pennell N, Combs D, Berinstein NL (2003) Pharmacokinetic study of patients with follicular or mantle cell lymphoma treated with rituximab as 'in vivo purge' and consolidative immunotherapy following autologous stem cell transplantation. Ann Oncol 14(5):758-765

29. Jager U, Fridrik M, Zeitlinger M, Heintel D, Hopfinger G, Burgstaller S, Mannhalter C, Oberaigner W, Porpaczy E, Skrabs C, Einberger C, Drach J, Raderer M, Gaiger A, Putman M, Greil 
R, Arbeitsgemeinschaft Medikamentose Tumortherapie I (2012) Rituximab serum concentrations during immuno-chemotherapy of follicular lymphoma correlate with patient gender, bone marrow infiltration and clinical response. Haematologica 97(9):14311438. https://doi.org/10.3324/haematol.2011.059246

30. Dirks NL, Meibohm B (2010) Population pharmacokinetics of therapeutic monoclonal antibodies. Clin Pharmacokinet 49(10):633659. https://doi.org/10.2165/11535960-000000000-00000

31. Anolik JH, Friedberg JW, Zheng B, Barnard J, Owen T, Cushing E, Kelly J, Milner EC, Fisher RI, Sanz I (2007) B cell reconstitution after rituximab treatment of lymphoma recapitulates B cell ontogeny. Clin Immunol 122(2):139-145. https://doi. org/10.1016/j.clim.2006.08.009

32. Dunleavy K, Hakim F, Kim HK, Janik JE, Grant N, Nakayama T, White T, Wright G, Kwak L, Gress R, Tosato G, Wilson WH (2005) B-cell recovery following rituximab-based therapy is associated with perturbations in stromal derived factor-1 and granulocyte homeostasis. Blood 106(3):795-802. https://doi.org/10.1182/ blood-2004-08-3198 